\title{
Generalized controllers for rigid formation stabilization with application to event-based controller design
}

\author{
$\begin{array}{llll}\text { Z. Sun } & \text { Q. Liu } & \text { C. Yu } & \text { B. D. O. Anderson }\end{array}$
}

\begin{abstract}
This paper discusses generalized controllers for rigid formation shape stabilization. We provide unified analysis to show convergence using different controllers reported in the literature, and further prove an exponential stability of the formation system when using the general form of shape controllers. We also show that different agents can use different controllers for controlling different distances to achieve a desired rigid formation, which enables the implementation of heterogeneous agents in practice for formation shape control. We further propose an event-triggered rigid formation control scheme based on the generalized controllers. The triggering condition, event function and convergence analysis are discussed.
\end{abstract}

\section{INTRODUCTION}

Formation control of networked multi-agent systems has received considerable attention in recent years due to its extensive applications. One problem of extensive interest is formation shape control, i.e. on designing controllers to maintain a geometrical shape for the formation [1]. By using rigid graph theory, the formation shape can be achieved by controlling a certain set of interagent distances [2], [3]. In the rigid formation stabilization problem, one typical controller that has been studied extensively in the literature takes the following form (see e.g. [3]):

$$
\dot{p}_{i}=\sum_{j \in \mathcal{N}_{i}}\left(p_{j}-p_{i}\right)\left(\left\|p_{j}-p_{i}\right\|^{2}-d_{i j}^{2}\right)
$$

where the definitions underpinning each notation will become clear in Section II. The above controller (1), which is derived from a well defined potential function, serves as a standard control law for stabilizing rigid formations. The dynamics of such gradient flow (1) have been investigated in several succeeding papers, e.g. [4]-[6]. We mention that alternative kinds of formation controllers other than the one in (1) are also available, which have been reported sparsely in the literature (see e.g. [7], [8]).

One of the aims in this paper is to propose and analyze general forms of formation controller to stabilize rigid shapes, and we provide a unified analysis to discuss the controller performance which generalizes most existing formation controllers in the literature. One key result in the controller analysis is an exponential stability of the formation system if some properties of the associated potential functions hold. The exponential stability of the formation control

Z. Sun and C. Yu are with Shandong Computer Science Center SCSC (National Supercomputer Center in Jinan), Shandong Provincial Key Laboratory of Computer Networks, Jinan, China, and B. D. O. Anderson is a visiting expert with SCSC. All the authors are with National ICT Australia and Research School of Engineering, The Australian National University, Canberra ACT 0200, Australia. (\{zhiyong.sun, qingchen.liu, brad.yu, brian.anderson\}eanu.edu.au) system has important consequences relating to robustness issues in rigid formations, as discussed in our previous papers [5], [9].

The second objective is to provide an event-based formation control strategy. This kind of controller design is attractive for real-world robots/vehicles equipped with digital sensors or microprocessors [10], [11]. Furthermore, by using an event-triggered mechanism to update the controller input, instead of using a continuous monitoring/updating strategy, the formation system can save resources in processors and thus can relax much computation/actuation burden for each agent. Due to these favourable properties, event-based controller has been studied extensively in recent years for linear or nonlinear systems [10], [11], and especially for distributed networked systems [12], [13] and multi-agent linear consensus systems [14], [15]. By extending the result of generalized controllers discussed in the first main part of this paper, we provide some first efforts on designing an event-based formation control scheme with the analysis dealing with triggering function and convergence.

The rest of this paper is organized as follows. In Section II, preliminary concepts on graph theory and rigidity theory are introduced. In Section III, we provide detailed analysis on generalized controllers and the associated exponential stability property. Section IV focuses on the event-based controller design based on generalized controllers. In Section V, some simulations are provided to demonstrate the controller performance. Finally, Section VI concludes this paper.

\section{BASIC CONCEPTS ON GRAPH AND RIGIDITY THEORY}

Consider an undirected graph with $m$ edges and $n$ vertices, denoted by $\mathcal{G}=(\mathcal{V}, \mathcal{E})$ with vertex set $\mathcal{V}=\{1,2, \cdots, n\}$ and edge set $\mathcal{E} \subset \mathcal{V} \times \mathcal{V}$. The neighbor set $\mathcal{N}_{i}$ of node $i$ is defined as $\mathcal{N}_{i}:=\{j \in \mathcal{V}:(i, j) \in \mathcal{E}\}$. The matrix relating the nodes to the edges is called the incidence matrix $H=$ $\left\{h_{i j}\right\} \in \mathbb{R}^{m \times n}$, whose entries are defined as (with arbitrary edge orientations for the undirected formations considered here)

$$
h_{i j}=\left\{\begin{array}{cc}
1, & \text { the } i \text {-th edge sinks at node } j \\
-1, & \text { the } i \text {-th edge leaves node } j \\
0, & \text { otherwise }
\end{array}\right.
$$

The adjacency matrix $A(\mathcal{G})$ is a symmetric $n \times n$ matrix encoding the vertex adjacency relationships, with entries $A_{i j}=1$ if $\{i, j\} \in \mathcal{E}$, and $A_{i j}=0$ otherwise. Another important matrix representation of a graph $\mathcal{G}$ is the Laplacian matrix $L(\mathcal{G})$, which is defined as $L(\mathcal{G})=H^{T} H=$ $\operatorname{diag}\left\{A \mathbf{1}_{n}\right\}-A$. For a connected undirected graph, one has 
$\operatorname{rank}(L)=n-1$ and $\operatorname{ker}(L)=\operatorname{ker}(H)=\operatorname{span}\left\{\mathbf{1}_{n}\right\}$. Note that for the rigid formation modelled by undirected graph considered in this paper, the orientation of each edge for writing the incidence matrix can be defined arbitrarily; the graph Laplacian matrix $L(\mathcal{G})$ for the undirected graph is always the same regardless of what edge orientations are defined (i.e. is orientation-independent) and the stability analysis in next sections remains unchanged.

Let $p_{i} \in \mathbb{R}^{d}$ where $d=\{2,3\}$ denote a point that is assigned to $i \in \mathcal{V}$. The stacked vector $p=$ $\left[p_{1}^{T}, p_{2}^{T}, \cdots, p_{n}^{T}\right]^{T} \in \mathbb{R}^{d n}$ represents the realization of $\mathcal{G}$ in $\mathbb{R}^{d}$. The pair $(\mathcal{G}, p)$ is said to be a framework of $\mathcal{G}$ in $\mathbb{R}^{d}$. By introducing the matrix $\bar{H}:=H \otimes I_{d} \in \mathbb{R}^{d m \times d n}$, one can construct the relative position vector as an image of $\bar{H}$ from the position vector $p$ :

$$
z=\bar{H} p
$$

where $z=\left[z_{1}^{T}, z_{2}^{T}, \cdots, z_{m}^{T}\right]^{T} \in \mathbb{R}^{d m}$, with $z_{k} \in \mathbb{R}^{d}$ being the relative position vector for the vertex pair defined by the $k$-th edge.

Using the same ordering of the edge set $\mathcal{E}$ as in the definition of $H$, the rigidity function $r_{\mathcal{G}}(p): \mathbb{R}^{d n} \rightarrow \mathbb{R}^{m}$ associated with the framework $(\mathcal{G}, p)$ is given as:

$$
r_{\mathcal{G}}(p)=\frac{1}{2}\left[\cdots,\left\|p_{i}-p_{j}\right\|^{2}, \cdots\right]^{T}, \quad(i, j) \in \mathcal{E}
$$

where the norm is the standard Euclidean norm, and the $k$-th component in $r_{\mathcal{G}}(p),\left\|p_{i}-p_{j}\right\|^{2}$, corresponds to the squared length of the relative position vector $z_{k}$ which connects the vertices $i$ and $j$.

The rigidity of frameworks is then defined as follows.

Definition 1: ( [16]) A framework $(\mathcal{G}, p)$ is rigid in $\mathbb{R}^{d}$ if there exists a neighborhood $\mathbb{U}$ of $p$ such that $r_{\mathcal{G}}^{-1}\left(r_{\mathcal{G}}(p)\right) \cap$ $\mathbb{U}=r_{\mathcal{K}}^{-1}\left(r_{\mathcal{K}}(p)\right) \cap \mathbb{U}$ where $\mathcal{K}$ is the complete graph with the same vertices as $\mathcal{G}$.

In the following, the set of all frameworks $(\mathcal{G}, p)$ which satisfies the distance constraints is referred to as the set of target formations. Let $\left(d_{k_{i j}}\right)$ denotes the desired distance in the target formation which links agent $i$ and $j$. We further define

$$
e_{k_{i j}}=\left\|p_{i}-p_{j}\right\|^{2}-\left(d_{k_{i j}}\right)^{2}
$$

to denote the squared distance error for edge $k$. (Note we will also use $e_{k}$ and $d_{k}$ occasionally for notational convenience if no confusion is expected.) Define the distance square error vector $e=\left[e_{1}, e_{2}, \cdots, e_{m}\right]^{T}$.

One useful tool to characterize the rigidity property of a framework is the rigidity matrix $R \in \mathbb{R}^{m \times d n}$, which is defined as

$$
R(p)=\frac{\partial r_{\mathcal{G}}(p)}{\partial p}
$$

It is not difficult to see that each row of the rigidity matrix $R$ takes the following form

$$
\left[\mathbf{0}_{1 \times d}, \cdots,\left(p_{i}-p_{j}\right)^{T}, \cdots, \mathbf{0}_{1 \times d}, \cdots,\left(p_{j}-p_{i}\right)^{T}, \cdots, \mathbf{0}_{1 \times d}\right]
$$

Each edge gives rise to a row of $R$, and, if an edge links vertices $i$ and $j$, then the nonzero entries of the corresponding row of $R$ are in the columns from $d i-(d-1)$ to $d i$ and from $d j-(d-1)$ to $d j$. The equation (2) shows that the relative position vector lies in the image of $\bar{H}$. Thus one can redefine the rigidity function, $g_{\mathcal{G}}(z): \operatorname{Im}(\bar{H}) \rightarrow \mathbb{R}^{m}$ as $g_{\mathcal{G}}(z)=\frac{1}{2}\left[\left\|z_{1}\right\|^{2},\left\|z_{2}\right\|^{2}, \cdots,\left\|z_{m}\right\|^{2}\right]^{T}$. From (2) and (4), one can obtain the following simple form for the rigidity matrix

$$
R(p)=\frac{\partial r_{\mathcal{G}}(p)}{\partial p}=\frac{\partial g_{\mathcal{G}}(z)}{\partial z} \frac{\partial z}{\partial p}=Z^{T} \bar{H}
$$

where $Z=\operatorname{diag}\left\{z_{1}, z_{2}, \cdots, z_{m}\right\}$.

The rigidity matrix will be used to determine the infinitesimal rigidity of the framework, as shown in the following definition.

Definition 2: ( [17]) A framework $(\mathcal{G}, p)$ is infinitesimally rigid in $d$-dimensional space if

$$
\operatorname{rank}(R(p))=d n-d(d+1) / 2
$$

Specifically, if the framework is infinitesimally rigid in $\mathbb{R}^{2}$ (resp. $\mathbb{R}^{3}$ ) and has exactly $2 n-3$ (resp. $3 n-6$ ) edges, then it is called a minimally and infinitesimally rigid framework. In this paper we focus on the stabilization problem of minimally and infinitesimally rigid formations. From the definition of infinitesimal rigidity, one can easily prove the following lemma:

Lemma 1: If the framework $(\mathcal{G}, p)$ is minimally and infinitesimally rigid in the $d$-dimensional space, then the matrix $R(p) R(p)^{T}$ is positive definite.

\section{CONVERGENCE ANALYSIS FOR GENERALIZED CONTROLLERS}

This section aims to provide a unified analysis for the convergence property when generalized controllers are constructed for stabilizing minimally rigid formations. One of the key results in this section is that exponential convergence can be derived for generalized controllers, thus most current results in the literature can be generalized.

Let us define a general potential function $V_{k}$ : $\left(-d_{k_{i j}}^{2}, \infty\right) \rightarrow \mathbb{R}_{\geq 0}$ with the following properties:

1) $V_{k}(\varphi)$ is nonnegative and continuously differentiable on $\left(-d_{k}^{2}, \infty\right)$;

2) $V_{k}(\varphi)$ and its gradient $g_{k}(\varphi):=\frac{\partial V_{k}(\varphi)}{\partial \varphi}$ are zero if and only if $\varphi=0$;

3) $g_{k}(\varphi)$ is strictly monotone increasing and continuously differentiable over its domain.

For each agent $i$, the local potential is constructed as $\Theta_{i}=$ $\frac{1}{2} \sum_{j \in \mathcal{N}_{i}} V_{k}\left(e_{k_{i j}}\right)$. According to the gradient control law, each agent takes the following gradient flow to minimize its local potential function:

$$
\dot{p}_{i}=-\nabla_{p_{i}} \Theta_{i}=-\sum_{j \in \mathcal{N}_{i}}\left(p_{i}-p_{j}\right) g_{k}\left(e_{k_{i j}}\right)
$$

Remark 1: The general potential function defined above generalizes many controls proposed in the literature. For 
example, in [7] the controller is a special form of (8) which takes $g_{k}\left(e_{k}\right)$ as

$$
g_{k}\left(e_{k}\right)=1-\frac{d_{k}}{\sqrt{\left(e_{k}+d_{k}^{2}\right)}}
$$

In [8], the designed controller involves a special form of $g_{k}\left(e_{k}\right)$ as

$$
g_{k}\left(e_{k}\right)=1-\frac{d_{k}^{2}}{\left(e_{k}+d_{k}^{2}\right)}
$$

In [18] and [19], the authors proposed a different potential function which generated the following $g_{k}\left(e_{k}\right)$

$$
g_{k}\left(e_{k}\right)=1-\frac{d_{k}^{4}}{\left(e_{k}+d_{k}^{2}\right)^{2}}
$$

Note that the above control functions (9), (10) and (11) have the attractive property of ensuring collision avoidance for the formation system.

The most commonly-used gradient control law for stabilizing rigid formations involves the potential function in the form of $V_{k}=1 / 2 e_{k}^{2}$, with the derived $g_{k}$ in a simple form:

$$
g_{k}\left(e_{k}\right)=e_{k}
$$

This controller was proposed in [3] and was further studied in e.g. [4]-[6].

Define a vector $\Phi$ to denote the gradients of the potential functions in each edge:

$$
\Phi(e)=\left[g_{1}\left(e_{1}\right), g_{2}\left(e_{2}\right), \cdots, g_{m}\left(e_{m}\right)\right]^{T}
$$

By noting that $\frac{\partial V_{k}\left(e_{k}\right)}{\partial p_{i}}=g_{k}\left(e_{k}\right) \frac{\partial e_{k}}{\partial p_{i}}$ and comparing the expression of the single agent system (8) with the rigidity matrix (5), one can write the following compact form of the overall controller:

$$
\dot{p}=-R^{T}(z) \Phi(e)
$$

where $R(z)$ is the rigidity matrix. Then there holds

$$
\begin{aligned}
\dot{e} & =\frac{\partial e}{\partial p} \dot{p}=2 \frac{\partial r_{\mathcal{G}}(p)}{\partial p} \dot{p}=2 Z^{T} \bar{H} \dot{p} \\
& =2 R(z) \dot{p}=-2 R(z) R^{T}(z) \Phi(e)
\end{aligned}
$$

Remark 2: Note that in the above system, the entries of the matrix $R(z)$ involve items of $z$. In [20] and [9], we have proved that when the formation shape is close to the desired one, the entries of the matrix $R(z) R^{T}(z)$ are continuously differentiable functions of $e$. So the system described in (14) is a self-contained system, and we will call it the distance error system in the sequel.

The main result in this section is stated in the following theorem which concerns an exponential convergence of the distance error vector $e$.

Theorem 1: By employing the gradient control (13) derived from the general potential functions, the distance error system (14) converges locally to zero exponentially fast.

Proof: We divide the whole proof into three main steps.

(I) Proof of asymptotic stability. Define the overall potential function: $V=\frac{1}{2} \sum_{k=1}^{m} V_{k}\left(\left\|z_{k}\right\|^{2}-d_{k}^{2}\right)$ which also serves as the candidate Lyapunov function. Further define a sub-level set $\Psi(\rho)=\{e: V(e) \leq \rho\}$ for some sufficiently small $\rho$, such that for all the points in the set $\Psi(\rho)$ the formation is infinitesimally minimally rigid and close to the target formation. Thus the rigidity matrix is of full row rank, and further $R(z) R^{T}(z)$ is positive definite. By calculating the derivative of $V$ along the trajectories of the distance error system (14), one can show

$$
\dot{V}(e)=-\Phi^{T}(e) R(z) R^{T}(z) \Phi(e)
$$

Further let $\lambda$ denote the minimum singular value of the rigidity matrix when $e$ is in the set $\Psi: \lambda=$ $\min _{e \in \Psi} e i g\left(R(z) R^{T}(z)\right)>0$. Note that the set $\Psi(\rho)$ is compact and the existence of such $\lambda$ is guaranteed. Then one has

$$
\dot{V}(e) \leq-\lambda\|\Phi(e)\|^{2}
$$

which indicates that $\dot{V}(e)$ is negative definite for $e \in \Psi \backslash\{0\}$. Thus the asymptotic stability of the equilibrium $e=0$ in the error system (14) is proved.

(II) Proof of two inequalities. To show the exponential convergence of the error system (14), it suffices to prove the following comparison inequalities (see e.g. Chapter 5.3 of [21]):

$$
\begin{gathered}
\|\Phi(e)\|^{2} \geq \varrho\|e\|^{2} \\
c_{1}\|e\|^{2} \leq V(e) \leq c_{2}\|e\|^{2}
\end{gathered}
$$

where $\varrho, c_{1}$ and $c_{2}$ are positive constants which need to be determined. The key properties to derive the above inequalities are that the gradient $g_{k}\left(e_{k}\right)$ is strictly monotone increasing and locally Lipschitz continuous (due to its continuous differentiability) on $\left(-d_{k}^{2}, \infty\right)$. We follow a similar approach as that in the previous paper [22] to show the above inequalities (17) and (18).

Recall the definition of the Lyapunov function candidate:

$$
V=\frac{1}{2} \sum_{k=1}^{m} V_{k}\left(\left\|z_{k}\right\|^{2}-d_{k}^{2}\right)=\frac{1}{2} \sum_{k=1}^{m} \int_{0}^{e_{k}} g_{k}(s) \mathrm{d} s
$$

Let $\epsilon$ be a positive number, which should satisfy

$$
\epsilon \leq \min \left\{d_{1}^{2}, d_{2}^{2}, \cdots, d_{m}^{2}\right\}
$$

We note that for all $e$ which satisfies $e \in \Psi(\rho) \backslash\{0\}$, such requirement for $\epsilon$ can be guaranteed.

Let

$$
\zeta_{k}=\inf _{|s| \leq \sqrt{\epsilon}} \frac{\mathrm{d} g_{k}(s)}{\mathrm{d} s}, \quad k \in\{1,2, \cdots, m\}
$$

Each $\zeta_{k}$ is positive because each $g_{k}$ is strictly monotone increasing on $\left(-d_{k}^{2}, \infty\right)$. From this and the fact that $g_{k}(0)=$ 0 it follows that

$$
\left|g_{k}(s)\right| \geq \zeta_{k}|s|,|s| \leq \sqrt{\epsilon}, \quad k \in\{1,2, \cdots, m\}
$$

Therefore

$$
\int_{0}^{e_{k}} g_{k}(s) \mathrm{d} s \geq \frac{\zeta_{k}}{2} e_{k}^{2}, \quad\left|e_{k}\right| \leq \sqrt{\epsilon}
$$

Choose

$$
2 c_{1}=\min \left\{\frac{\zeta_{1}}{2}, \frac{\zeta_{2}}{2}, \cdots, \frac{\zeta_{m}}{2}\right\}
$$


One has $V(e) \geq c_{1}\|e\|^{2}$. Then the left inequality of (18) is true.

Since each $g_{k}$ is Lipschitz continuous and satisfies $g_{k}(0)=$ 0 , there exist positive constants $\delta_{k}$ such that $\left|g_{k}(s)\right| \leq \delta_{k}|s|$ for $|s| \leq \sqrt{\epsilon}$. Then it follows that

$$
\int_{0}^{e_{k}} g_{k}(s) \mathrm{d} s \leq \frac{\delta_{k}}{2} e_{k}^{2}, \quad\left|e_{k}\right| \leq \sqrt{\epsilon}
$$

Choose

$$
2 c_{2}=\max \left\{\frac{\delta_{1}}{2}, \frac{\delta_{2}}{2}, \cdots, \frac{\delta_{m}}{2}\right\}
$$

One has $V(e) \leq c_{2}\|e\|^{2}$. Then the right part of (18) is proved.

From (21) it is obvious that $\left|g_{k}\left(e_{k}\right)\right|^{2} \geq \zeta_{k}^{2}\left|e_{k}\right|^{2}$. By choosing

$$
\zeta^{2}=\min \left\{\zeta_{1}^{2}, \zeta_{2}^{2}, \cdots, \zeta_{m}^{2}\right\}
$$

one has $\|\Phi(e)\|^{2} \geq \zeta^{2}\|e\|^{2}$. Let $\varrho=\zeta^{2}$, which proves the inequality in (17).

(III) Proof of exponential stability. This step is standard from the results of the above two steps. By combining the inequalities in (16), (17) and (18), one obtains that

$$
\dot{V}(e) \leq-\lambda \varrho\|e\|^{2} \leq-\frac{\lambda \varrho}{c_{2}} V(e)
$$

which indicates the following

$$
\|e(t)\| \leq\left(\frac{c_{2}}{c_{1}}\right)^{\frac{1}{2}} e^{-\gamma t}\|e(0)\|
$$

with the exponential convergence rate $\gamma=\frac{\lambda \varrho}{2 c_{2}}$.

We show some intrinsic properties of the formation system with the generalized controllers.

Lemma 2: The formation stabilization system designed in (8) (and in the compact form (13)) with the generalized controller has the following properties:

1) The controller is decentralized and each agent requires only relative position measurements of its neighboring agents.

2) The center of the mass of the formation is stationary.

3) The controller for each agent is independent of any global coordinates. That is, each agent can use its own coordinate system to measure the necessary relative positions and to implement the control.

The proof is omitted due to space limit and will be shown in the full version of this paper.

Remark 3: We mention here three significant aspects of the above result stated in Theorem 1. First, it generalizes many existing controllers in a unified form (e.g. [3], [4], [7], [8], [18], [19]) and for the first time it proves the exponential convergence for such kind of generalized controllers with certain properties. Second, the exponential stability obtained from the generalized controller also has important robustness consequences in case of distance perturbations, which extends the robustness behaviors in formation systems observed in [5] and [9], where the controllers considered in [5] and [9] were confined to be the one of (12). Third, we note that for different agents to stabilize each distance, the function $g_{k}$ in each controller can take different forms. This enables the concept of heterogeneous agents for implementing formation control strategy as each agent may have different sensing/controlling performances reflected by different $g_{k}$.

\section{EVENT-BASED CONTROLLER DESIGN}

The controller design using the event based idea is motivated by the fact that event-based controllers can alleviate the requirements of continuous sensor measurement or controller actuation, via discrete-time measurements or actuation in controllers. In this section we provide some preliminary analysis on event-based controller design by extending the results from the above section. We consider the following general form of the event-triggered controller

$$
\begin{aligned}
\dot{p}_{i}(t) & =u_{i}(t)=u\left(t_{h}\right) \\
& \left.=\sum_{j \in \mathcal{N}_{i}}\left(p_{j}(t)-p_{i}(t)\right) g_{k}\left(e\left(t_{h}\right)\right)\right)
\end{aligned}
$$

for $t \in\left[t_{h}, t_{h+1}\right]$, where $t_{h}$ is the last triggering time for updating new information. The basic problem here is to design event triggering times and the specific events involved in the control function $u_{i}(t)$.

To deal with the position system with the event-triggered controller (29), we instead analyze first the distance error system. Via similar steps shown in (14), the error system with event-triggered controller can be derived as

$$
\dot{e}=-2 R(t) R^{T}(t) \Phi\left(e\left(t_{h}\right)\right)
$$

Denote a vector $\delta(t)=e\left(t_{h}\right)-e(t), h=0,1,2, \cdots$ for $t \in\left[t_{h}, t_{h+1}\right]$. The self-contained error system can be written as

$$
\dot{e}=-2 R(t) R^{T}(t) \Phi(\delta(t)+e(t))
$$

The system (30) or (31) can be considered as a switching system. Consider the function $V=\frac{1}{2} \sum_{k=1}^{m} V_{k}\left(\left\|z_{k}\right\|^{2}-d_{k}^{2}\right)$ as a Lyapunov-like function candidate. Similar to the proof in Theorem 1, we define a sub-level set $\Psi_{2}(\rho)=\{e$ : $V(e) \leq \rho\}$ for some sufficiently small $\rho$, such that when $e \in \Psi_{2}(\rho)$ the formation is infinitesimally minimally rigid and $R(z) R^{T}(z)$ is positive definite. Then one can show that

$$
\begin{aligned}
\dot{V} & =\frac{1}{2} \Phi^{T}(e) \dot{e}=-\Phi^{T}(e) R(z) R^{T}(z) \Phi(\delta(t)+e(t)) \\
& \leq-e^{T} \Gamma R(z) R^{T}(z) \Gamma(\delta(t)+e(t)) \\
& \leq-\lambda_{\min }(M)\|e\|^{2}+\|\delta\|\|M\|\|e\|
\end{aligned}
$$

where $\Gamma=\operatorname{diag}\left\{\zeta_{1}, \zeta_{2}, \cdots, \zeta_{m}\right\}$, and $M=\Gamma R(z) R^{T}(z) \Gamma$. The inequality in the second line of (32) is derived by (21). Note that $M$ is positive definite since $R R^{T}$ is positive definite and $\Gamma$ is a diagonal matrix with positive diagonal entries, hence $\lambda_{\min }(M)$ is well defined and is positive.

The norm of $\delta$ is enforced to satisfy

$$
\|\delta\| \leq \gamma \frac{\lambda_{\min }(M)\|e\|}{\|M\|}
$$

where we choose the parameter $\gamma$ to satisfy that $0<\gamma<1$, and thus guarantee that

$$
\dot{V} \leq(\delta-1) \lambda_{\min }(M)\|e\|^{2}<0 .
$$


This indicates that we can design the triggering event as

$$
f(e):=\|\delta\|-\gamma \frac{\lambda_{\min }(M)\|e\|}{\|M\|}=0
$$

and the event time is defined as $f\left(e\left(t_{h}\right)\right)=0$ for $h=$ $0,1,2, \cdots$. For the time interval $t \in\left[t_{h}, t_{h+1}\right]$, the control input is chosen as $u(t)=u\left(t_{h}\right)$ until the next event is triggered. Furthermore, every time when an event is triggered, the event vector $\delta$ will be reset to zero.

Theorem 2: If the initial formation is close to the target formation, by using the above controller (29) and the eventtriggering function (34), all the agents will reach the desired formation shape exponentially fast.

In the following we will further show that the eventtriggered switching system (29) does not show Zeno behavior.

Lemma 3: The inter-event time interval $\left\{t_{h+1}-t_{h}\right\}$ is lower bounded by a positive value $\tau$

$$
\tau=\frac{\gamma \bar{\lambda}_{\min }(M)}{\|M\|\left(\|M\|+\gamma \bar{\lambda}_{\min }(M)\right)}
$$

where $\bar{\lambda}_{\text {min }}(M)$ denotes the smallest value of $\lambda_{\min }(t)$ in the convergence process.

The proof is inspired by the one used in [23] (and further in [14]) and is omitted here due to the space limit.

We also show a key property of the shape stabilization system with the above event-triggered controller.

Lemma 4: The formation centroid remains constant under the control of (29).

The proof is omitted here due to space limit.

Remark 4: The event-based controller for rigid formation stabilization in this section is a preliminary one and several improvements can be considered. One direction is to design feasible event-based controllers such that the adjustment of direction and magnitude can be updated in discrete time and the requirement of continuous measurement in the trigger function can be relaxed (via. e.g. a self-triggered scheme). The other direction is to design a fully decentralized controller, in which each agent is equipped with an event trigger and can trigger its own control output by using local information from its neighbors. The challenge in the decentralized design arises because the formation controller is nonlinear, the matrix $R$ is state dependent, and the global information $\lambda_{\min }$ is required. We note that $\lambda_{\min }$ can be estimated by each agent in a distributed way, by using the discrete-time eigenvalue estimation technique in [24] which is inspired by [25] and [26]. These are on-going topics which will be reported elsewhere.

\section{Simulation Results}

In this section we provide two simulations to show the behavior of certain formations with generalized controllers. Firstly consider a 4-agent formation shape, with the desired distances given as $d_{12}^{*}=d_{34}^{*}=3, d_{23}^{*}=d_{14}^{*}=4$, $d_{13}^{*}=5$ corresponding to a rectangular shape. The initial conditions for each agent are chosen as $p_{1}(0)=[0,0]^{T}$, $p_{2}(0)=[-1,4]^{T}, p_{3}(0)=[5,3]^{T}$ and $p_{4}(0)=[3,0]^{T}$,

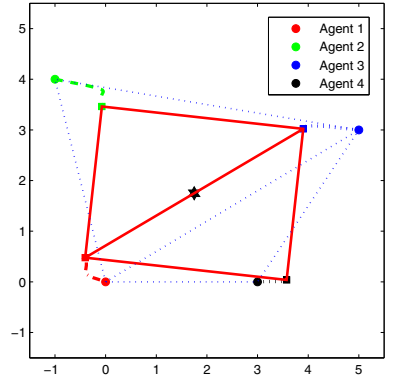

Fig. 1. Simulation on an 4-agent rectangular formation shape. The initial and final positions are denoted by circles and squares, respectively. The initial formation is denoted by dotted blue lines, and the final formation is denoted by red solid lines. The black star denotes the formation centroid.

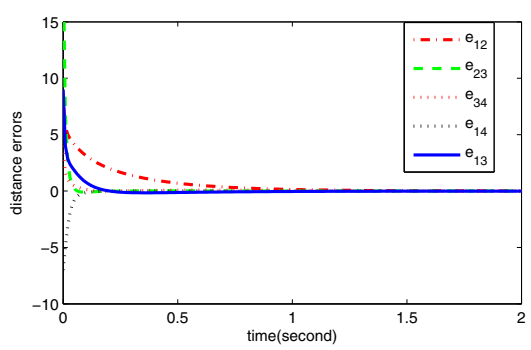

Fig. 2. Exponential convergence of the distance errors.

so that the initial formation is close to the target one. We assume that for different edges the agents are using different controller forms. Specifically, for the edges $\{12\}$ and $\{34\}$ the controller contributions in the related agents are based on (10), for the edges $\{12\}$ and $\{13\}$ the controller contributions are based on (9), and for edges $\{23\}$ and $\{14\}$ the agents use the form of (12). The trajectories of each agent and the final shape are depicted in Fig. 1. The trajectories of each distance error are depicted in Fig. 2, which shows an exponential convergence to the origin.

We then perform simulations on the event-based controller. We suppose the same simulation settings as above, but
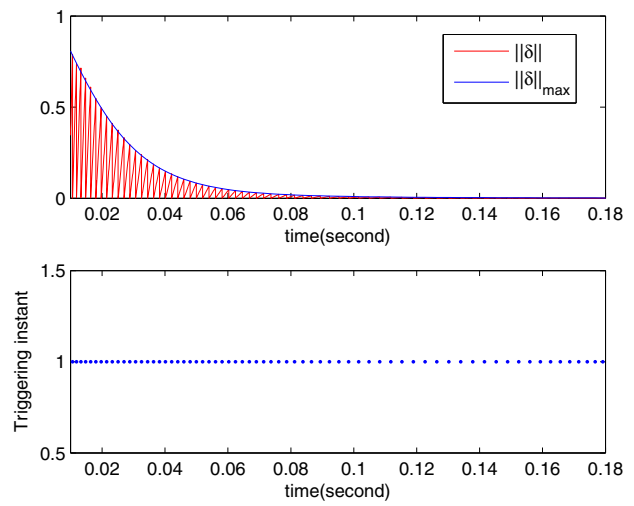

Fig. 3. Performance of the event-based controller. Top: evolution of $\|\delta\|$ and $\|\delta\|_{\max }=\gamma \frac{\lambda_{\min }(M)\|e\|}{\|M\|}$. Bottom: event triggering instants 


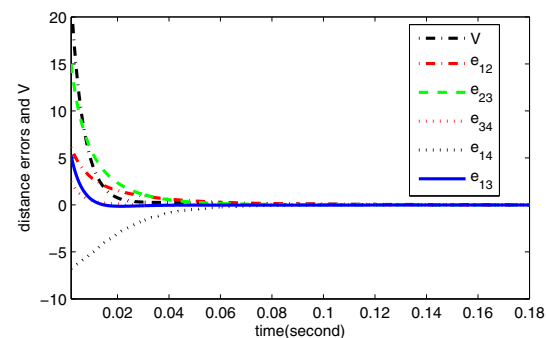

Fig. 4. Exponential convergence of the distance errors and Lyapunov function with the event-based controller

assume each controller involves $g_{k}=e_{k}$ for the simplicity of demonstration. The parameter $\gamma$ in the trigger function is set as $\gamma=0.9$. Fig. 3 shows the evolution of the norm of the vector $\delta$ in the trigger function (34), which is obviously bounded below by $\gamma \frac{\lambda_{\min }(M)\|e\|}{\|M\|}$ as required by (33). Fig. 4 indicates the exponential convergence of each distance error and the Lyapunov function.

\section{CONCLUSIONS}

In this paper we have shown general results for a unified form of shape controllers to stabilize rigid formations, which has generalized most controllers with different forms in the literature (e.g. [3]-[8], [18], [19]). One of the main results shows an exponential convergence property of the formation system with the generalized controller which also enables an implementation with heterogenous agents in formation control. We have further shown how to design an eventbased controller for rigid formation stabilization, which also shows an exponential convergence while no Zeno behavior will exist. Future research topics include global convergence analysis of the formation system with the generalized controllers by using the technique of e.g., [22], [27], and further relaxation of the requirements of the centralized processing and continuous measurement in the trigger function. The design of a distributed event-triggered controller for rigid formation control will be reported elsewhere.

\section{ACKNOWLEDGMENT}

This work was supported by NICTA, which is funded by the Australian Government as represented by the Department of Broadband, Communications and the Digital Economy and the Australian Research Council (ARC) through the ICT Centre of Excellence program. B. D. O. Anderson and C. Yu were also supported by the ARC under grant DP130103610. Z. Sun is also supported by the Prime Minister's Australia Asia Incoming Endeavour Postgraduate Award.

\section{REFERENCES}

[1] K.-K. Oh, M.-C. Park, and H.-S. Ahn, "A survey of multi-agent formation control," Automatica, vol. 53, pp. 424-440, 2015.

[2] B. D. O. Anderson, C. Yu, B. Fidan, and J. M. Hendrickx, "Rigid graph control architectures for autonomous formations," Control Systems Magazine, IEEE, vol. 28, no. 6, pp. 48-63, 2008.

[3] L. Krick, M. E. Broucke, and B. A. Francis, "Stabilisation of infinitesimally rigid formations of multi-robot networks," International Journal of Control, vol. 82, no. 3, pp. 423-439, 2009.
[4] F. Dorfler and B. Francis, "Geometric analysis of the formation problem for autonomous robots," Automatic Control, IEEE Transactions on, vol. 55, no. 10, pp. 2379-2384, 2010.

[5] A. Belabbas, S. Mou, A. S. Morse, and B. D. O. Anderson, "Robustness issues with undirected formations," in Proc. of the 51st Conference on Decision and Control, pp. 1445-1450, 2012.

[6] B. D. O. Anderson and U. Helmke, "Counting critical formations on a line," SIAM Journal on Control and Optimization, vol. 52, no. 1, pp. 219-242, 2014.

[7] B. D. O. Anderson, C. Yu, S. Dasgupta, and A. S. Morse, "Control of a three-coleader formation in the plane," Systems \& Control Letters, vol. 56, no. 9, pp. 573-578, 2007.

[8] S. L. Smith, M. E. Broucke, and B. A. Francis, "Stabilizing a multiagent system to an equilateral polygon formation," in Proc. of the 17th International Symposium on Mathematical Theory of Networks and Systems, pp. 2415-2424, 2006.

[9] Z. Sun, S. Mou, B. D. O. Anderson, and A. S. Morse, "Nonrobustness of gradient control for 3-D undirected formations with distance mismatch," in Proc. of the 3rd Australian Control Conference, pp. 369-374, IEEE, 2013.

[10] K. J. Aström, "Event based control," in Analysis and design of nonlinear control systems, pp. 127-147, Springer, 2008.

[11] W. Heemels, K. H. Johansson, and P. Tabuada, "An introduction to event-triggered and self-triggered control.," in Proc. of the 51st Conference on Decision and Control, pp. 3270-3285, 2012.

[12] M. Mazo and P. Tabuada, "Decentralized event-triggered control over wireless sensor/actuator networks," IEEE Transactions on Automatic Control, vol. 56, no. 10, pp. 2456-2461, 2011.

[13] D. V. Dimarogonas, E. Frazzoli, and K. H. Johansson, "Distributed event-triggered control for multi-agent systems," IEEE Transactions on Automatic Control, vol. 57, no. 5, pp. 1291-1297, 2012.

[14] D. V. Dimarogonas and K. H. Johansson, "Event-triggered cooperative control," in Proc. of the 2009 European Control Conference, pp. 30153020, 2009.

[15] G. S. Seyboth, D. V. Dimarogonas, and K. H. Johansson, "Event-based broadcasting for multi-agent average consensus," Automatica, vol. 49, no. 1, pp. 245-252, 2012.

[16] L. Asimow and B. Roth, "The rigidity of graphs, II," Journal of Mathematical Analysis and Applications, vol. 68, no. 1, pp. 171-190, 1979.

[17] B. Hendrickson, "Conditions for unique graph realizations," SIAM Journal on Computing, vol. 21, no. 1, pp. 65-84, 1992.

[18] D. V. Dimarogonas and K. H. Johansson, "On the stability of distancebased formation control," in Proc. of the 47th Conference on Decision and Control, pp. 1200-1205, IEEE, 2008.

[19] Y. Tian and Q. Wang, "Global stabilization of rigid formations in the plane," Automatica, vol. 49, no. 5, pp. 1436-1441, 2013.

[20] S. Mou, A. S. Morse, M. A. Belabbas, Z. Sun, and B. D. O. Anderson, "Undirected rigid formations are problematic," IEEE Transactions on Automatic Control, conditionally accepted, 2014.

[21] S. Sastry, Nonlinear systems: analysis, stability, and control, vol. 10. Springer New York, 1999.

[22] M. Cao, A. S. Morse, C. Yu, B. D. O. Anderson, and S. Dasgupta, "Maintaining a directed, triangular formation of mobile autonomous agents," Communications in Information and Systems, vol. 11, no. 1, pp. 1-16, 2011.

[23] P. Tabuada, "Event-triggered real-time scheduling of stabilizing control tasks," IEEE Transactions on Automatic Control, vol. 52, no. 9, pp. 1680-1685, 2007.

[24] Z. Sun, C. Yu, and B. D. O. Anderson, "Distributed estimation and control for preserving formation rigidity for mobile robot teams," arXiv preprint arXiv:1309.4850, pp. 1-8, 2013.

[25] R. Aragues, G. Shi, D. V. Dimarogonas, C. Sagues, and K. H Johansson, "Distributed algebraic connectivity estimation for adaptive event-triggered consensus," in Proc. of the 2012 American Control Conference, pp. 32-37, IEEE, 2012.

[26] D. Zelazo, A. Franchi, H. H. Bülthoff, and P. R. Giordano, "Decentralized rigidity maintenance control with range-only measurements for multi-robot systems," International Journal of Robotics Research, also available at arXiv:1309.0535, 2013.

[27] M.-C. Park, Z. Sun, B. D. O. Anderson, and H.-S. Ahn, "Stability analysis on four agent tetrahedral formations," Proc. of the 53rd IEEE Conference on Decision and Control (CDC 2014), pp. 631-636, 2014. 